\title{
Bevacizumab in the neoadjuvant treatment of human epidermal growth factor receptor 2-negative breast cancer: A meta-analysis of randomized controlled trials
}

\author{
ZEINA NAHLEH ${ }^{1}$, GEHAN BOTRUS $^{2}$, ALOK DWIVEDI $^{3}$, \\ MICHAEL JENNINGS ${ }^{4}$, SHAIMAA NAGY ${ }^{5}$ and ARAFAT TFAYLI ${ }^{6}$
}

\begin{abstract}
${ }^{1}$ Department of Hematology-Oncology, Maroone Cancer Center, Cleveland Clinic Florida, Weston, FL 33331; Departments of ${ }^{2}$ Internal Medicine and ${ }^{3}$ Biomedical Sciences, Texas Tech University Health Sciences Center; ${ }^{4}$ Texas Tech University Health Sciences Center, Paul L. Foster School of Medicine, El Paso, TX 79912, USA; ${ }^{5}$ Department of Pathology, Faculty of Medicine, Benha University, Benha 13511, Egypt;

${ }^{6}$ American University of Beirut Medical Center, Beirut 1107-2020, Lebanon
\end{abstract}

Received October 1, 2018; Accepted December 20, 2018

DOI: $10.3892 /$ mco.2019.1796

\begin{abstract}
Several randomized clinical trials have suggested the effectiveness of bevacizumab (Bev) in early and advanced breast cancer; however, due to the increased toxicity and lack of a clear long-term survival benefit, there is currently no defined role for Bev in breast cancer in the USA, while it has been approved in Europe. We herein sought to conduct a meta-analysis of large randomized trials comparing the efficacy and long-term outcome of neoadjuvant chemotherapy with Bev compared with chemotherapy without Bev in human epidermal factor receptor 2 (HER2)-negative breast cancer. A search was conducted through PubMed and Ovid Medline databases. Among the 279 articles identified, 5 met the eligibility criteria and were included in the present analysis. A total of 2,268 patients treated with Bev and 2,278 treated without Bev were analyzed. Pathological complete response (pCR) was obtained in $35 \%$ of patients treated with Bev and in $26 \%$ of those treated without Bev. A statistically significant increase (26\%) in the incidence of pCR was observed in the Bev-treated group. However, patients treated with Bev exhibited no significant difference in the risk of disease recurrence or death. To the best of our knowledge, this is the first meta-analysis addressing the long-term outcomes of Bev in combination with chemotherapy in the neoadjuvant treatment of HER2-negative breast cancer. The results confirmed the significant benefit of Bev combined with chemotherapy compared with chemotherapy alone on breast cancer response, in both triple-negative and hormone receptor-positive cases. However, this benefit does
\end{abstract}

Correspondence to: Dr Zeina Nahleh, Department of Hematology-Oncology, Maroone Cancer Center, Cleveland Clinic Florida, 2950 Cleveland Clinic Blvd, Weston, FL 33331, USA E-mail: nahlehz@ccf.org

Key words: HER2 negative, breast cancer, bevacizumab, neoadjuvant not translate into a long-term disease-free or definitive overall survival advantage. Optimizing patient selection is desirable for maximizing the long-term benefits of Bev, while reducing cost and treatment-related adverse effects. Future efforts directed toward the discovery of predictive markers would be crucial for identifying the subset(s) of breast cancer patients who are most likely to benefit from Bev therapy.

\section{Introduction}

Breast cancer is the most commonly diagnosed invasive cancer in women and the second most common cause of cancer-related mortality (1). In 2016, 246,660 new cases of invasive breast cancer were diagnosed in the USA, with an estimated 40,000 deaths (1). Significant advances in the understanding and therapeutic approaches to breast cancer have been made over the past several decades, which have led to improved patient outcomes and decreased breast cancer-related mortality (2). Historically, neoadjuvant therapy has been reserved for locally advanced disease as a means of reducing the tumor burden and increasing the efficacy of surgical resection (3). The National Surgical Adjuvant Breast and Bowel Project (NSABP) B-18 trial was one of the first to demonstrate that neoadjuvant therapy is as effective as adjuvant therapy in terms of disease-free survival (DFS) and overall survival (OS), and it provides the added benefit of increasing the rate of breast-conserving surgery (4). In recent years, drug development in the neoadjuvant setting has become an important research focus, allowing for a potentially more rapid investigation of tumor biology and predictive markers (3-9). Bevacizumab (Bev) was the first vascular endothelial growth factor (VEGF)-A inhibitor to be studied in breast cancer and other solid cancers (10-14), and is currently approved by the USA Food and Drug Administration (FDA) for use in several cancer types (12-14). Following provisional approval for use in metastatic breast cancer in the USA, the FDA subsequently revoked approval in November 2011, due to the risks of Bev therapy outweighing the benefits $(15,16)$. Currently, Bev is only approved for the treatment of breast 
cancer outside the USA. In the neoadjuvant setting, while the effects of Bev on increasing the rate of pathological complete response (pCR) are well-documented, there is currently no clear evidence on the effects of Bev on long-term outcome, or its side effects. Despite the potential benefits of Bev as adjuvant therapy, it is not approved for human epidermal growth factor receptor (HER)2-negative breast cancer patients due to its side effects. However, no study has yet compared all side effects between Bev vs. non-Bev groups. A recent meta-analysis (17) was undertaken to determine the effect of Bev on pCR in patients with HER2-negative breast cancer. However, that study was based on mixed randomized and non-randomized trials and included heterogeneous populations; it also did not evaluate the long-term effects or adverse effects of Bev compared with non-Bev. In view of the controversy surrounding Bev, and given the efficacy of Bev in early and advanced breast cancer but the lack of a clearly defined role in this disease, we sought to conduct a meta-analysis of large, high-quality randomized trials to compare the effect of neoadjuvant chemotherapy with and without Bev on HER2-negative breast cancer. The aim was to determine whether the achievement of higher rates of $\mathrm{pCR}$ with combined neoadjuvant chemotherapy and Bev compared with chemotherapy alone is associated with improved long-term survival.

\section{Materials and methods}

Search strategy. We systematically searched for any phase II or III randomized clinical trials evaluating the effect of Bev combined with chemotherapy in the neoadjuvant setting for early or locally advanced breast cancer. The PubMed and Ovid Medline databases were searched between January 1990 and August 2016, using the search terms 'breast' AND 'neoadjuvant OR preoperative' AND 'bevacizumab OR avastin' AND 'randomized OR randomised OR random OR RCT OR trial'. After an initial search, the articles were reviewed and only large $(n \geq 200)$ prospective trials on HER2-negative breast cancer were included in this meta-analysis. The PRISMA guidelines were followed for reporting and conducting this meta-analysis $(18,19)$. G. Botrus and A. Dwivedi independently analyzed the studies eligible for inclusion. Any disagreements between the two reviewers were resolved by consulting a senior investigator (Z. Nahleh).

Inclusion and exclusion criteria. Articles were included in the present analysis based on the following critieria: i) Randomized controlled trials; ii) evaluating neoadjuvant chemotherapy treatment with and without Bev; iii) including stage I-III breast cancer; iv) HER2-negative and v) providing data on pCR, DFS and OS. Articles were excluded if they were animal studies, not written in English, based on endocrine therapy rather than chemotherapy, not published as full manuscripts, not including Bev, not randomized controlled trials, and including stage IV breast cancer cases.

Outcome measures and data extraction. The primary endpoints for this meta-analysis were $\mathrm{pCR}, \mathrm{pCR}$ in estrogen receptor (ER)/progesterone receptor $(\mathrm{PgR})$-positive cancer, and pCR in triple-negative breast cancer (TNBC). The secondary endpoints were OS, DFS and adverse events. The data extracted included year of publication, study endpoints such as pCR, pCR in ER/PgR-positive cases, pCR in triple-negative cases, DFS, OS and any-grade adverse events. Additional data on demographics or clinical characteristics were also recorded.

Statistical analysis. The incidence and relative risk (RR) for the outcome measures and standard error (SE) for each study were compared between Bev and non-Bev. Different studies used different statistics to summarize time-to-event outcomes, such as OS and DFS. In such cases, the hazard ratio (HR) and SE of HR were computed using the suggested methods (20). To obtain the pooled estimate, inverse variance weights were computed for each study. Inverse variance weight adds more weight to more precise studies. After computing incidence, RR, HR, SE and weight of each outcome for each study, the heterogeneity in the studies was evaluated. $\mathrm{I}^{2}$ was used to evaluate heterogeneity in the studies. $\mathrm{I}^{2}>70 \%$ was considered to reflect substantial heterogeneity. The fixed-effects or random-effects model was used to compute the combined effect of Bev compared with non-Bev from different studies. When significant heterogeneity was present, the random-effects model (DerSimonian and Laird method) was used for estimating the RR/HR of outcomes in Bev compared with non-Bev. The combined effect size was summarized using either overall incidence or RR/HR, along with $95 \%$ confidence interval (CI). All analyses were conducted using Stata software, v.13.0 (StataCorp LP, College Station, TX, USA). $\mathrm{P}<0.05$ was considered to indicate a statistically significant difference.

\section{Results}

Study selection. The search through electronic databases using different combinations of search terms produced 279 articles. The flow chart of the study selection process (Fig. 1) illustrates the identification of eligible articles at different review stages. A total of 5 randomized studies were ultimately included in this meta-analysis (5,21-25). A total of 4,526 patients (2,268 treated with Bev and 2,278 without Bev) were included in the analysis. The characteristics of the individual studies are summarized in Table I. The overall incidence of the outcomes considered in the present study and the pooled effect of Bev compared with non-Bev on pCR are shown in Table II.

Comparison of pCR between the Bev and non-Bev groups. The rate of pCR was estimated as 30\% (95\% CI: 21-40\%), irrespective of the treatment groups (pooled estimate). The pCR range was $18-52 \%$ (21-59\% in Bev vs. $17-48 \%$ in the non-Bev group), and the overall incidence of pCR was $35 \%$ with Bev vs. $26 \%$ without Bev. A relative increase of $26 \%$ in the incidence of pCR was observed in the Bev-treated group compared with the non-Bev group $(\mathrm{RR}=1.26,95 \% \mathrm{CI}$ : $1.15-1.38, \mathrm{P}<0.001)$. As shown in Fig. 2, all the studies favored Bev for pCR compared with non-Bev, without significant heterogeneity $\left(\mathrm{I}^{2}=0 \%\right)$. The addition of Bev to chemotherapy in the neoadjuvant setting significantly increased the rate of pCR (breast and lymph nodes) $(\mathrm{RR}=1.22,95 \% \mathrm{CI}$ : $1.10-1.36, \mathrm{P}<0.001)$ compared with treatment without Bev. In TNBC, there was a $30 \%$ relative increase in the incidence of $\mathrm{pCR}(\mathrm{RR}=1.30,95 \% \mathrm{CI}: 1.16-1.45$, $\mathrm{P}<0.001)$ in patients treated with Bev vs. the non-Bev group. In addition, in $\mathrm{ER} / \mathrm{PgR}+$ cases, there was a $27 \%$ relative increase 


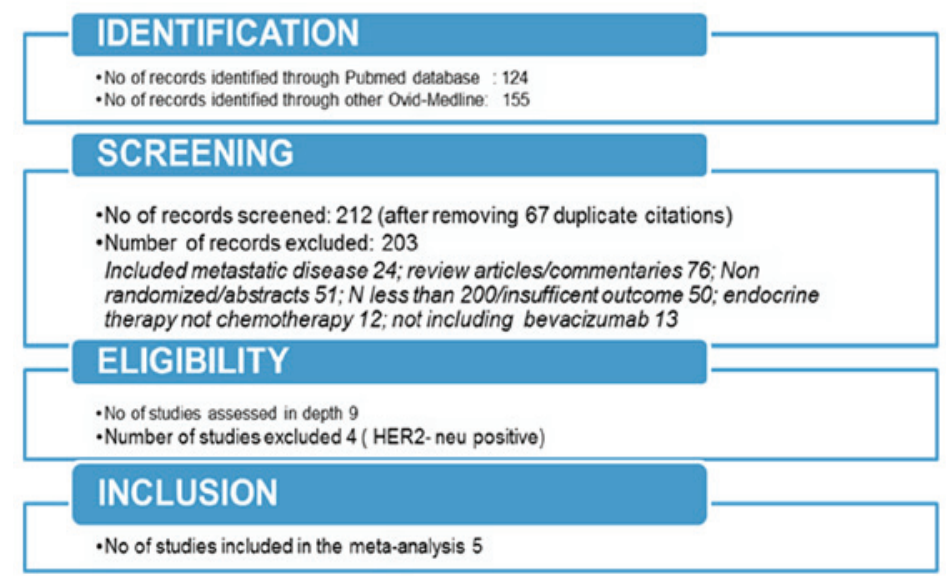

Figure 1. Study selection process. HER2, human epidermal growth factor receptor 2.

in the incidence of $\mathrm{pCR}(\mathrm{RR}=1.27,95 \% \mathrm{CI}: 1.13-1.42, \mathrm{P}<0.001)$ in patients treated with Bev compared with the non-Bev group (Table III).

Comparison of OS and DFS between the Bev and non-Bev groups. Of the 5 studies analyzed, 4 reported the DFS and OS status by treatment groups (Figs. 3 and 4). Patients treated with Bev exhibited no difference in the risk of recurrence $\left(\mathrm{I}^{2}=35.7 \%\right.$; HR=0.97, 95\% CI: 0.85-1.11, $\left.\mathrm{P}=0.684\right)$ without presence of significant heterogeneity $(\mathrm{P}=0.198)$; however, a trend towards reducing the risk of death $\left(\mathrm{I}^{2}=64 \% ; \mathrm{HR}=0.90\right.$; 95\% CI: 0.76-1.06, $\mathrm{P}=0.194)$ was noted, without substantial heterogeneity in the effect $\left(\mathrm{I}^{2}=64, \mathrm{P}=0.04\right)$.

Comparison of adverse events between the Bev and non-Bev groups. The incidence of grade 3 and 4 adverse events was compared between treatment groups and the results are summarized in Table IV. Individual reported adverse events were compared across studies. Some adverse events were found to be significantly more common in the Bev group compared with the non-Bev group, including hypertension ( $R=5.36)$, mucositis $(R R=5.23)$, peripheral neuropathy $(R R=1.75)$, febrile neutropenia $(R R=1.71)$, infection $(R R=1.68)$, hand-foot syndrome $(\mathrm{R}=1.57)$ and neutropenia $(\mathrm{R}=1.06)$.

\section{Discussion}

To the best of our knowledge, this is the first meta-analysis addressing the long-term outcomes of Bev in combination with chemotherapy in the neoadjuvant treatment of HER2-negative breast cancer. The addition of Bev to chemotherapy was found to significantly increase the incidence of pCR (breast and lymph nodes) compared with treatment without Bev in $\mathrm{ER} / \mathrm{PgR}$-positive and triple-negative cases. Although the addition of Bev to chemotherapy did not translate into a significant difference in DFS, it exhibited a trend towards improving OS, but without reaching statistical significance. Despite the heterogeneity in reporting adverse events among the trials, there was no significant difference in the incidence of grade 3 and 4 adverse events between the Bev and non-Bev groups. However, patients treated with Bev more commonly experienced hypertension, mucositis, peripheral neuropathy and neutropenia compared with those treated without Bev. These findings are consistent with the adverse events associated with the use of Bev, as the most common grade 3/4 adverse events include hypertension followed by mucositis.

The results of the present study are consistent with the findings from another recent meta-analysis that included 9 studies and reported the efficacy of Bev with chemotherapy compared with Bev without chemotherapy in terms of pCR as the primary endpoint of interest, without assessing long-term survival outcome (17). However, there were notable differences between the two studies: Cao et al (17), combined studies with both HER2-negative and HER2-positive breast cancer and included mostly preliminary findings, without examining the long-term effects of Bev. By contrast, the present analysis included completed randomized trials, excluded HER2-positive breast cancer, and reported long-term survival data. As regards to efficacy, we also observed some differences. The overall efficacy of Bev in terms of pCR in HER2-negative patients was found to be slightly lower in our analysis, which had a larger sample size, compared with the one reported by Cao et al (17).

The results of our analysis were consistent with the reported outcome on $\mathrm{pCR}$ in the 5 neoadjuvant trials considered (5,21-25). However, there were also certain variations. In our analysis, an overall relative increase of $26 \%$ in the incidence of pCR was observed in the Bev group compared with the non-Bev group, which is very similar to the pCR reported in the studies analyzed (5,21-23). One study (24) that included primarily higher-risk patients with locally advanced and inflammatory breast cancer (S0800) reported a slightly higher RR of pCR in the Bev group compared with the non-Bev group, as opposed to the other 4 studies and the pooled effect obtained in this study. The variation observed in the S0800 study may be due to its comparatively smaller size relative to the other 4 studies considered in this meta-analysis.

A notable difference in our analysis was that the benefit of Bev treatment in increasing pCR rate was observed in both the triple-negative (30\% increase in relative incidence of pCR, $11 \%$ absolute benefit) as well as in the ER/PgR-positive groups (26\% increase in relative incidence of pCR, $9 \%$ absolute 


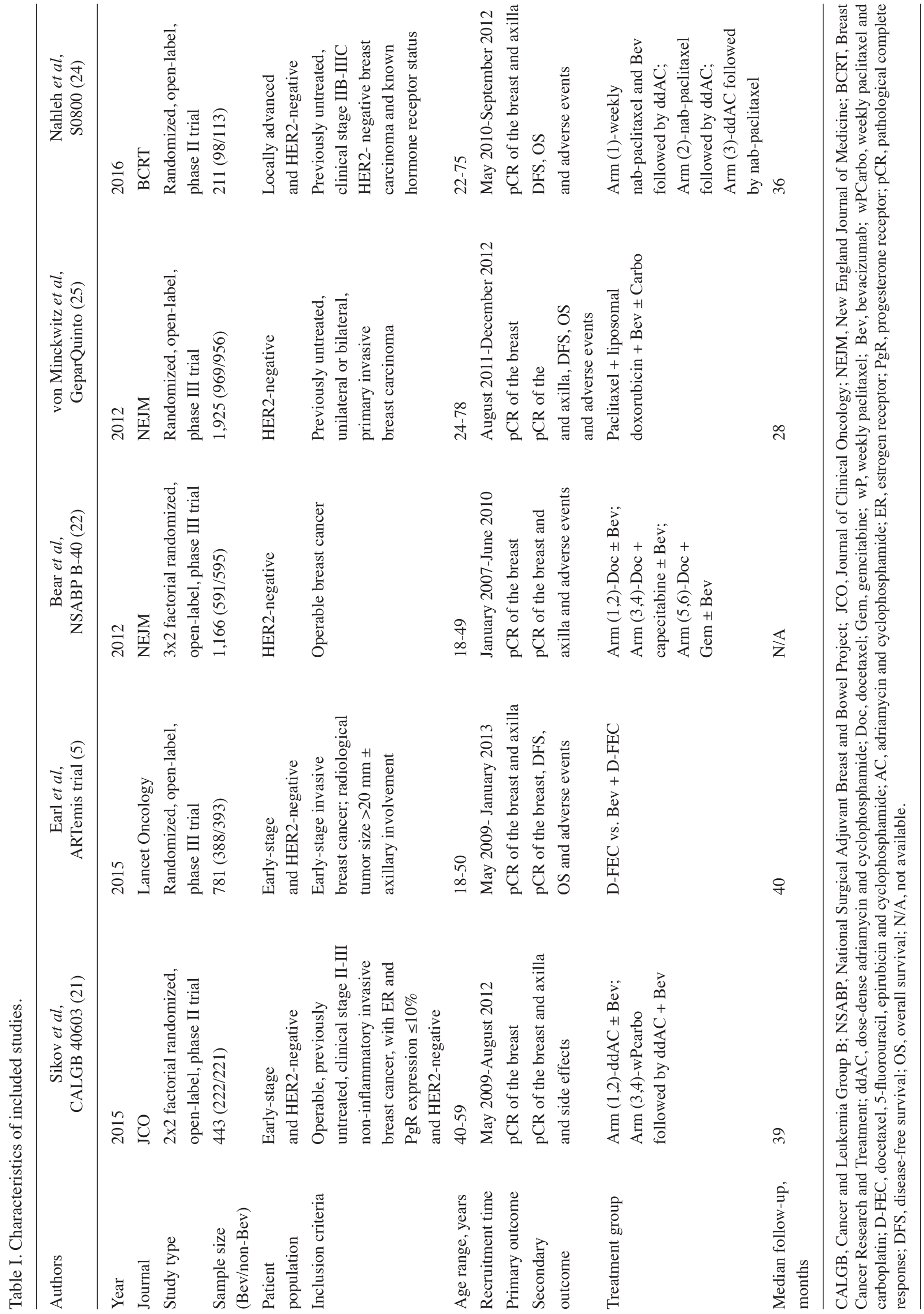


Table II. Pooled estimate of pCR by subgroups.

\begin{tabular}{|c|c|c|c|c|}
\hline & & $\begin{array}{l}\text { Overall } \\
\text { incidence }\end{array}$ & \multicolumn{2}{|c|}{$95 \%$ CI } \\
\hline $\mathrm{pCR}$ & 98.1 & 0.30 & 0.21 & 0.40 \\
\hline $\begin{array}{l}\text { In breast }+ \\
\text { lymph nodes }\end{array}$ & 98.1 & 0.27 & 0.17 & 0.36 \\
\hline $\begin{array}{l}\text { In } \mathrm{ER} / \mathrm{PgR} \mathrm{R}^{+} \\
\text {cases }\end{array}$ & 0.0 & 0.50 & 0.48 & 0.52 \\
\hline In TNBC & 95.3 & 0.53 & 0.44 & 0.62 \\
\hline
\end{tabular}

benefit). The ARTemis trial (5) reported a $6 \%$ adjusted absolute increase in the incidence of pCR after addition of Bev to standard neoadjuvant therapy compared with the non-Bev group (26 vs. $19 \%$ overall (95\% CI: $0.1-12.1)$; $\mathrm{P}=0.02$ ), but the efficacy was restricted to the ER-negative/HER-2-negative subgroup defined as Allred 0-2 (15\% absolute improvement) and the ER weakly positive group (Allred 3-5) (20\% absolute improvement), whereas there was no benefit in the ER strongly positive group (Allred 6-8) (absolute decrease of 1\%). Similarly, in the GeparQuinto study (25), the addition of Bev significantly increased the incidence of pCR in TNBC (11.4\% absolute benefit), in contrast to only $0.1 \%$ in the hormone receptor-positive subgroup. The CALGB 40603 study (21), a randomized phase II trial limited to stage II-III TNBC reported a significant increase in pCR incidence in the Bev group compared with the non-Bev group (59 vs. $48 \%$, respectively; $\mathrm{P}=0.009)$. The $\mathrm{S} 0800$ trial (24) demonstrated a significant increase in pCR rate with the addition of Bev in both the ER/PgR-positive and triple-negative groups, but the increase only reached statistical significance in the TNBC group. The findings of the NSABP B-40 trial (22) were somewhat contradictory, showing a significant increase in the number of patients achieving $\mathrm{pCR}$ in the hormone-positive group ( 23.2 vs. $15.1 \%, \mathrm{P}=0.007)$ but not in the triple-negative group (51.5 vs. $47.1 \%, \mathrm{P}=0.34)$.

The apparent discordance between the various studies may be explained by differences in study design and definitions of hormone receptor-positive disease. An ER/PgR-positive disease was defined as $>10 \%$ positive cells for either ER or PgR in GeparQuinto (25) and CALGB 40603 (21), while a $1 \%$ cutoff was used in S0800 (24) and NSABP B-40 (22), and an Allred score $>2$ was used in ARTemis trial to identify ER/PgR-positive patients (5), a definition similar to that of NSABP B-40 and S0800. It is, therefore, plausible that the increased proportion of patients who achieved a pCR in the ER/PgR-positive group in NSABP B-40 and S0800 resulted from patients with low ER/PgR scores who were excluded by GeparQuinto and CALGB 40603. Despite these differences, however, our analysis suggests that the benefits from Bev may not be limited to TNBC cases.
Table III. Pooled estimate of pCR in Bev compared with non-Bev.

\begin{tabular}{|c|c|c|c|c|c|}
\hline \multirow[b]{2}{*}{$\mathrm{pCR}$} & \multirow{2}{*}{$\frac{\mathrm{I}^{2}}{0.0}$} & \multirow{2}{*}{$\begin{array}{l}\mathrm{RR} \\
1.26\end{array}$} & \multicolumn{2}{|c|}{$95 \%$ CI } & \multirow{2}{*}{$\frac{\text { P-value }}{<0.001}$} \\
\hline & & & 1.15 & 1.38 & \\
\hline $\begin{array}{l}\text { In breast }+ \\
\text { lymph nodes }\end{array}$ & 0.0 & 1.22 & 1.10 & 1.36 & $<0.001$ \\
\hline $\begin{array}{l}\text { In } \mathrm{ER} / \mathrm{PgR}^{+} \\
\text {cases }\end{array}$ & 8.9 & 1.27 & 1.13 & 1.42 & $<0.001$ \\
\hline In TNBC & 0.0 & 1.30 & 1.16 & 1.45 & $<0.001$ \\
\hline
\end{tabular}

CI, confidence interval; $\mathrm{I}^{2}$, measure of heterogeneity; pCR, pathological complete response; ER, estrogen receptor; $\mathrm{PgR}$, progesterone receptor; TNBC, triple-negative breast cancer.

Our meta-analysis failed to demonstrate that the addition of Bev in the neoadjuvant setting improved DFS. This was not unexpected, considering the small absolute increment noted in pCR (11\% for TNBC and $9 \%$ for ER/PgR-positive cases). This finding is consistent with the results reported from the 5 considered studies. The association between pCR and event-free survival (EFS) has been quite challenging to elucidate (26). Berry and Hudis analyzed the potential causes for the discordance between pCR and EFS (26). They noted the difficulty to demonstrate a significant correlation when the treatment effect is being measured based on a small number of clinical trials or trial subsets, particularly given the small treatment differences in pCR rates in the trials and the inherent variability in reassembling patients into trials and treatment arms. However, the neoadjuvant approach to future drug development may still have a potentially significant role, should future phase 3 trials implement specific novel adaptive designs, such as the use of interim by-treatment information regarding $\mathrm{pCR}$ and EFS and adapting to accumulating information in the trial, among other strategies (26).

In our analysis, the small change in pCR rates combined with the different combinations of chemotherapy regimens used, different disease stages, discontinuation of Bev after surgery and possible rebound angiogenesis (27), as well as the varied median follow-up periods, would be plausible explanations for the lack of effect regarding DFS. However, the overall effect of Bev on OS exhibited a positive trend favoring Bev compared with non-Bev. This positive effect on OS was largely attributed to two trials $(22,24)$, while a lack of effect of Bev on OS was reported in one trial (5).

The results of the present meta-analysis are consistent with two large phase III postoperative trials indicating absence of a DFS and OS advantage from Bev when used in HER2-negative breast cancer $(28,29)$. The E5103 trial recruited 4,994 patients with HER2-negative disease and both hormone receptor-positive and -negative disease (29), while the BEATRICE trial enrolled 2,591 patients with only TNBC (28). In both trials, Bev was added to chemotherapy. After completing chemotherapy, the patients were randomly assigned to receive or not receive single-agent Bev for a total duration of 1 year. Neither trial showed an improvement in DFS or OS with the addition of 1 year of Bev to the adjuvant 


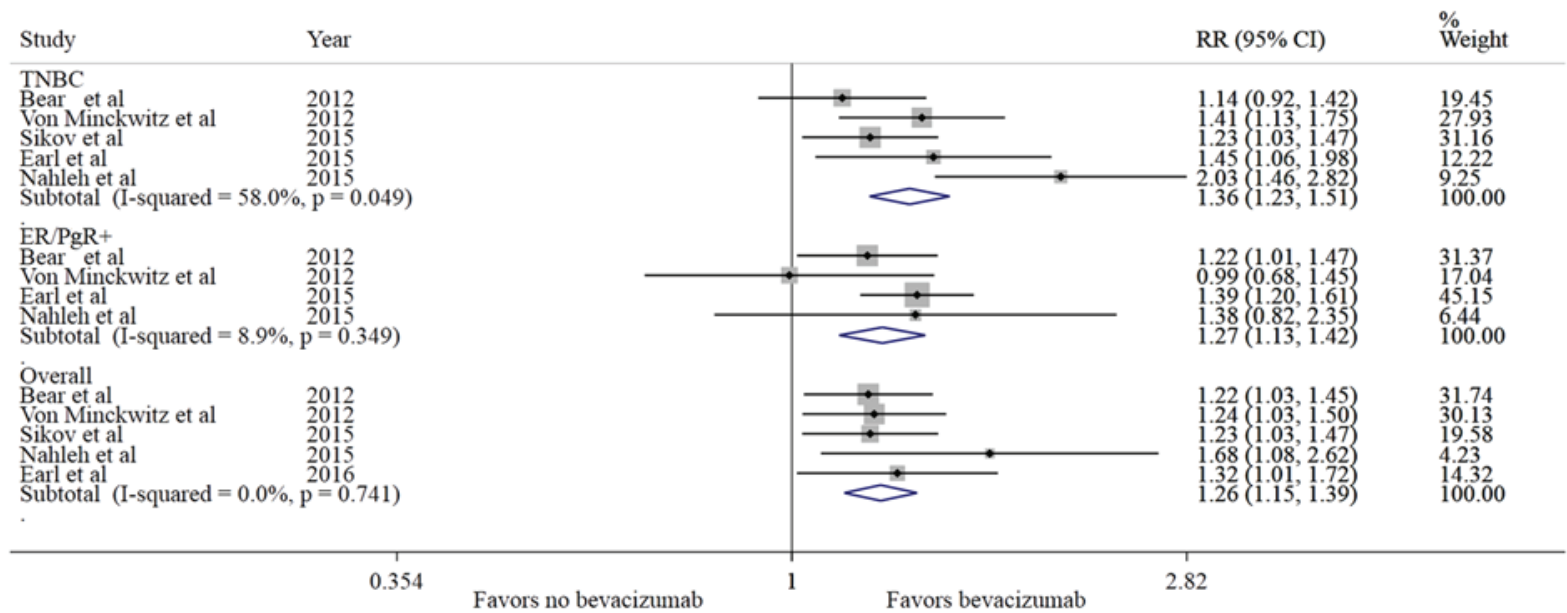

Figure 2. Comparison of pCR between Bev vs. no Bev groups. pCR, pathological complete response; Bev, bevacizumab; RR, relative risk; CI, confidence interval; TNBC, triple-negative breast cancer; ER, estrogen receptor; PgR, progesterone receptor.

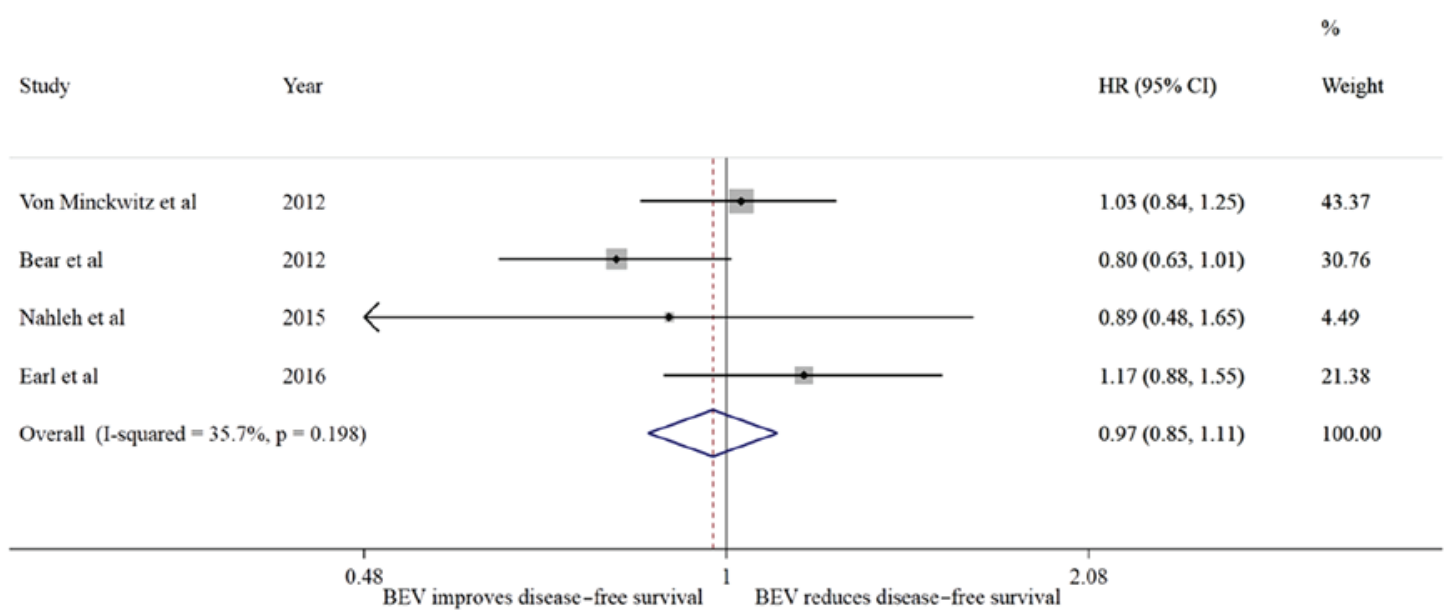

Figure 3. Comparison of DFS between Bev vs. non-Bev groups. DFS, disease-free survival; Bev, bevacizumab; HR, hazard ratio; CI, confidence interval.

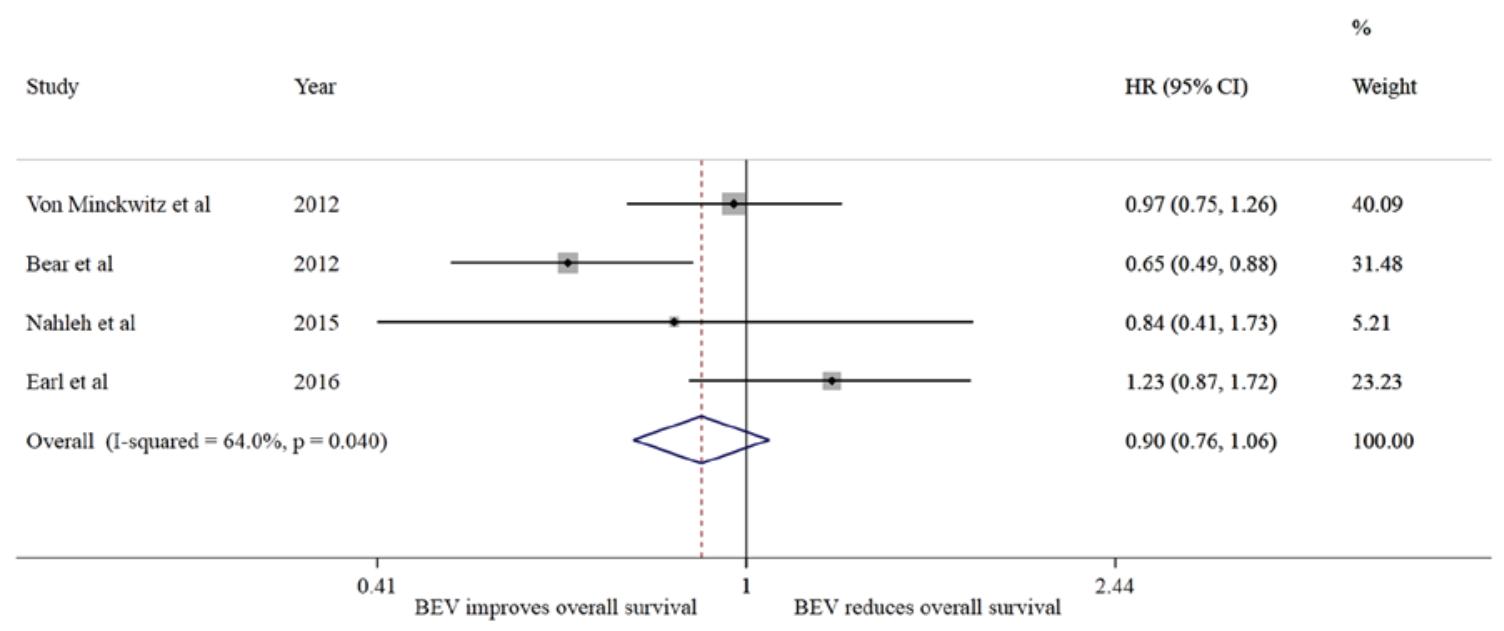

Figure 4. Comparison of OS between Bev vs. no Bev groups. OS, overall survival; Bev, bevacizumab; HR, hazard ratio; CI, confidence interval.

chemotherapy. Thus, the addition of continuous Bev treatment after surgery cannot be fully supported by the outcome of those trials. On the other hand, the positive trend in OS favoring Bev compared with chemotherapy without Bev noted in our analysis requires further investigation. In light of the NSABP-B40 data, it may be hypothesized that the benefit of $\mathrm{Bev}$ is most pronounced when used in a combined pre- and postoperative setting. When used as such, Bev, a targeted 
Table IV. Risk of grade3/4 adverse events in Bev compare with non-Bev.

\begin{tabular}{lccccr}
\hline & & & \multicolumn{2}{c}{$95 \%$ CI } & \\
\cline { 5 - 5 } Adverse events & $\mathrm{I}^{2}$ & $\mathrm{RR}$ & & & P-value \\
\cline { 5 - 6 } Grade 1/2 & 0.0 & 1.00 & 0.94 & 1.06 & 0.915 \\
Grade 3/4 & 93.9 & 1.14 & 0.90 & 1.44 & 0.280 \\
Leukopenia & 0 & 1.02 & 0.74 & 1.41 & 0.882 \\
Neutropenia & 37.5 & 1.06 & 1.01 & 1.11 & 0.024 \\
Thrombocytopenia & 4 & 1.05 & 0.61 & 1.81 & 0.849 \\
Hemoglobin & 50.9 & 1.32 & 0.70 & 2.50 & 0.392 \\
Febrile & 50.9 & 1.71 & 1.39 & 2.11 & $<0.001$ \\
neutropenia & & & & & \\
Nausea & 0 & 0.91 & 0.65 & 1.30 & 0.613 \\
Vomiting & 73.4 & 0.80 & 0.53 & 1.21 & 0.293 \\
Mucositis & 72.6 & 5.23 & 3.70 & 7.40 & $<0.001$ \\
Diarrhea & 50.1 & 0.84 & 0.60 & 1.18 & 0.316 \\
Hypertension & 74.1 & 5.36 & 3.51 & 8.19 & $<0.001$ \\
Peripheral & 3.2 & 1.75 & 1.086 & 2.81 & 0.021 \\
neuropathy & & & & & \\
Fatigue & 0 & 1.20 & 0.95 & 1.52 & 0.136 \\
Pain & 22.4 & 1.11 & 0.71 & 1.73 & 0.665 \\
Infection & 25.2 & 1.68 & 1.35 & 2.09 & $<0.001$ \\
Thrombosis & 0 & 1.43 & 0.91 & 2.24 & 0.119 \\
Hand-foot syndrome & 0 & 1.57 & 1.04 & 2.38 & 0.034 \\
\hline
\end{tabular}

CI, confidence interval; $\mathrm{I}^{2}$, measure of heterogeneity; RR, relative risk; Bev, bevacizumab.

anti-angiogenic agent, may be most useful in treating relatively large or more locally advanced tumors in the breast and lymph nodes, where high dependence on angiogenesis may be at play. This activity is reflected by the increased $\mathrm{pCR}$ with the use of Bev in the present meta-analysis, and particularly in trials including locally advanced and inflammatory tumors, such as the S0800 (24). The continuation of Bev after surgery may provide an additional benefit by suppressing possible rebound tumor cell growth (27).

As noted above, there was some heterogeneity observed for reporting adverse events across the different studies. In the CALGB 40603 study (21), 12\% of patients administered Bev, with or without carboplatin, stopped treatment early due to toxicity. Similarly, $14 \%$ of patients receiving Bev in the ARTemis (5) trial and 12\% of patients in the GeparSixto trial (23) stopped treatment early due to the toxicity. The most common grade $3 / 4$ adverse events included hypertension followed by mucositis, peripheral neuropathy, febrile neutropenia, infection and hand-foot syndrome, and the incidence was increased with Bev compared with non-Bev. Other studies have also demonstrated that Bev use is associated with increased incidence of grade 3 and 4 hypertension and mucositis. However, an increase in grade 3 and 4 left ventricular systolic dysfunction, thromboembolic events, bleeding and postoperative complications with Bev was not reported in the individual studies included in our analysis $(5,7,21,23-25)$.
One of the major limitations in the clinical applicability of $\mathrm{Bev}$ and potentially other similar agents in breast cancer is the current lack of information regarding specific prognostic markers correlated with Bev efficacy. Prognostic markers for Bev therapy have been studied in other solid cancers, and it has been suggested that improved efficacy of Bev in metastatic colorectal cancer is correlated with specific endothelial nitric oxide synthase (eNOS) polymorphisms (30). The greatest improvement in progression-free survival was associated with the haplotype homozygous for eNOS VNTR 4bb and eNOS + 894 TT. Various VEGFR1 and VEGFA single-nucleotide polymorphisms (SNPs) have also been implicated as prognostic markers for Bev therapy $(31,32)$. The recent findings of the ANGIOMET study demonstrated that VEGFA SNP rs3025039 and VEGFR1 SNP rs9582036 were associated with reduced OS in patients treated with Bev for non-small-cell lung cancer (32). Lower basal VEGF levels were also associated with better patient outcomes. This is in agreement with earlier reports suggesting that high levels of VEGF are correlated with increased vascular count and density, which may result in greater tumor growth (33). In the CALGB 40603 trial, the investigators studied how intrinsic subtype assigned by PAM50 and other gene signatures affected the impact of Bev on pCR rates in TNBC (34). A significant benefit of Bev was noted in the basal-like subtype, yielding a more than double the odds of achieving $\mathrm{pCR}$. In basal-like tumors, pCR was $64 \%$ with Bev vs. $45 \%$ without Bev (odds ratio $=2.15, \mathrm{P}=0.0009$ ). However, for the other subtypes, the rates were 43 and $60 \%$, respectively (odds ratio $=0.50, \mathrm{P}=0.25$ ), indicating a possible trend toward worse outcome when Bev was added (33). Furthermore, higher levels of expression of any of the following immune signatures, mRNA signatures for high proliferative rate, low estrogen signaling, high TP53 mutation, and an increase in the number of tumor-infiltrating lymphocytes, were associated with higher pCR rates. High proliferation rate, low estrogen expression, or high TP53-mutation signatures, in particular, were predictive of higher $\mathrm{pCR}$ rates overall, and a greater $\mathrm{pCR}$ benefit from the addition of Bev. These findings suggest that, even within TNBC, there are biologically defined patient subsets that may benefit from this agent. Further research is necessary, however, to confirm and validate reliable predictive and prognostic markers of Bev efficacy for the treatment of breast cancer.

In summary, the present meta-analysis confirms the benefit of Bev combined with chemotherapy compared with chemotherapy alone in the neoadjuvant treatment of HER2-negative breast cancer. This benefit in pCR is quite intriguing, as it does not translate into a long-term DFS or definitive OS advantage. Therefore, in the absence of specific predictive or efficacy markers, the use of non-targeted $\mathrm{Bev}$ in the treatment of an unselected breast cancer patient population cannot be justified at this time. This is particularly true in view of the adverse effect profile associated with the use of Bev. Further research is warranted for the pre-treatment identification of predictive and prognostic markers to identify the subsets of breast cancer patients who are most likely to benefit from Bev therapy. However, the premature dismissal of this effective agent in the treatment armamentarium of breast cancer is not recommended. Should a breast cancer patient subset be identified that may benefit from the addition of Bev, the studies included in this analysis can be revisited and confirmatory studies conducted using collected stored tissue samples. Furthermore, predictors of the efficacy of Bev, if identified in ongoing or future translational studies, may prompt new trials of this drug in better defined patient subgroups. 


\section{Acknowledgements}

Not applicable.

\section{Funding}

No funding was received.

\section{Availability of data and materials}

The datasets generated and analyzed during the present study are available from the corresponding author on reasonable request.

\section{Ethics approval and consent to participate}

Not applicable.

\section{Patient consent for publication}

Not applicable.

\section{Authors' contributions}

$\mathrm{ZN}$ developed the concept and design of the present study, collected the data and wrote and edited manuscript. GB collected data and contributed to the writing of the manuscript. AD developed the statistical method and performed data analysis. MJ collected data and SN contributed to data collection. AT wrote the manuscript performed the final revision. All the authors read and approved the final version of this manuscript.

\section{Competing interests}

The authors declare that they have no competing interests.

\section{References}

1. Howlader N, Noone AM, Krapcho M, Miller D, Bishop K, Altekruse SF, Kosary CL, Yu M, Ruhl J, Tatalovich Z, Mariotto A, Lewis DR, Chen HS, Feuer EJ, Cronin KA (eds). SEER Cancer Statistics Review, 1975-2013. National Cancer Institute, Bethesda MD, USA, 2016

2. DeSantis CE, Fedewa SA, Goding Sauer A, Kramer JL, Smith RA and Jemal A: Breast cancer statistics, 2015: Convergence of incidence rates between black and white women. CA Cancer J Clin 66: 31-42, 2016.

3. Gampenrieder SP, Rinnerthaler G and Greil R: Neoadjuvant chemotherapy and targeted therapy in breast cancer: Past, present, and future. J Oncol 2013: 732047, 2013.

4. Fisher B, Brown A, Mamounas E, Wieand S, Robidoux A, Margolese RG, Cruz AB Jr, Fisher ER, Wickerham DL, Wolmark $\mathrm{N}$, et al: Effect of preoperative chemotherapy on local-regional disease in women with operable breast cancer: Findings from national surgical adjuvant breast and bowel project B-18. J Clin Oncol 15: 2483-2493, 1997.

5. EarlHM,HillerL,DunnJA,BlenkinsopC,GrybowiczL, Vallier AL, Abraham J, Thomas J, Provenzano E, Hughes-Davies L, et al; ARTemis Investigators: Efficacy of neoadjuvant bevacizumab added to docetaxel followed by fluorouracil, epirubicin, and cyclophosphamide, for women with HER2-negative early breast cancer (ARTemis): An open-label, randomised, phase 3 trial. Lancet Oncol 16: 656-666, 2015.

6. Huober J, von Minckwitz G, Denkert C, Tesch H, Weiss E, Zahm DM, Belau A, Khandan F, Hauschild M, Thomssen C, et al: Effect of neoadjuvant anthracycline-taxane-based chemotherapy in different biological breast cancer phenotypes: Overall results from the GeparTrio study. Breast Cancer Res Treat 124: 133-140, 2010
7. Gerber B, Loibl S, Eidtmann H, Rezai M, Fasching PA, Tesch H, Eggemann H, Schrader I, Kittel K, Hanusch C, et al; German Breast Group Investigators: Neoadjuvant bevacizumab and anthracycline-taxane-based chemotherapy in 678 triple-negative primary breast cancers; results from the geparquinto study (GBG 44). Ann Oncol 24: 2978-2984, 2013.

8. Levasseur N, Clemons M, Hilton J, Addison C, Robertson S, Ibrahim $\mathrm{M}$ and Arnaout A: Neoadjuvant endocrine therapy and window of opportunity trials: New standards in the treatment of breast cancer? Minerva Chir 70: 181-193, 2015

9. Dowsett M: Predictive and prognostic factors. Breast Cancer Res 12 (Suppl 4): S2, 2010.

10. Shinkaruk S, Bayle M, Laïn G and Déléris G: Vascular endothelial cell growth factor (VEGF), an emerging target for cancer chemotherapy. Curr Med Chem Anticancer Agents 3: 95-117, 2003.

11. Ferrara N, Hillan KJ, Gerber HP and Novotny W: Discovery and development of bevacizumab, an anti-VEGF antibody for treating cancer. Nat Rev Drug Discov 3: 391-400, 2004.

12. Summers J, Cohen MH, Keegan P and Pazdur R: FDA drug approval summary: Bevacizumab plus interferon for advanced renal cell carcinoma. Oncologist 15: 104-111, 2010.

13. Gil-Gil MJ, Mesia C, Rey M and Bruna J: Bevacizumab for the treatment of glioblastoma. Clin Med Insights Oncol 7: 123-135, 2013.

14. Cohen MH, Gootenberg J, Keegan P and Pazdur R: FDA drug approval summary: Bevacizumab (Avastin) plus Carboplatin and Paclitaxel as first-line treatment of advanced/metastatic recurrent nonsquamous non-small cell lung cancer. Oncologist 12: 713-718, 2007.

15. Conti RM, Dusetzina SB, Herbert AC, Berndt ER, Huskamp HA and Keating NL: The impact of emerging safety and effectiveness evidence on the use of physician-administered drugs: The case of bevacizumab for breast cancer. Med Care 51: 622-627, 2013.

16. Lenzer J: FDA committee votes to withdraw bevacizumab for breast cancer. BMJ: doi.org/10.1136/bmj.d4244.

17. Cao L, Yao GY, Liu MF, Chen LJ, Hu XL and Ye CS: Neoadjuvant bevacizumab plus chemotherapy versus chemotherapy alone to treat non-metastatic breast cancer: A meta-analysis of randomised controlled trials. PLoS One 10: e0145442, 2015.

18. Moher D, Liberati A, Tetzlaff J and Altman DG; PRISMA Group: Preferred reporting items for systematic reviews and meta-analyses: The PRISMA statement. BMJ: doi.org/10.1136/ bmj.b2535.

19. Hutton B, Moher D and Cameron C: The PRISMA extension statement. Ann Intern Med 163: 566-567, 2015.

20. Tierney JF, Stewart LA, Ghersi D, Burdett S and Sydes MR: Practical methods for incorporating summary time-to-event data into meta-analysis. Trials 8: 16, 2007.

21. Sikov WM, Berry DA, Perou CM, Singh B, Cirrincione CT, Tolaney SM, Kuzma CS, Pluard TJ, Somlo G, Port ER, et al: Impact of the addition of carboplatin and/or bevacizumab to neoadjuvant once-per-week paclitaxel followed by dose-dense doxorubicin and cyclophosphamide on pathologic complete response rates in stage II to III triple-negative breast cancer: CALGB 40603 (Alliance). J Clin Oncol 33: 13-21, 2015.

22. Bear HD, Tang G, Rastogi P, Geyer CE Jr, Robidoux A, Atkins JN, Baez-Diaz L, Brufsky AM, Mehta RS, Fehrenbacher L, et al: Bevacizumab added to neoadjuvant chemotherapy for breast cancer. N Engl J Med 366: 310-320, 2012.

23. von Minckwitz G, Schneeweiss A, Loibl S, Salat C, Denkert C, Rezai M, Blohmer JU, Jackisch C, Paepke S, Gerber B, et al: Neoadjuvant carboplatin in patients with triple-negative and HER2-positive early breast cancer (GeparSixto; GBG 66): A randomised phase 2 trial. Lancet Oncol 15: 747-756, 2014.

24. Nahleh ZA, Barlow WE, Hayes DF, Schott AF, Gralow JR, Sikov WM, Perez EA, Chennuru S, Mirshahidi HR, Corso SW, et al: SWOG S0800 (NCI CDR0000636131): Addition of bevacizumab to neoadjuvant nab-paclitaxel with dose-dense doxorubicin and cyclophosphamide improves pathologic complete response (pCR) rates in inflammatory or locally advanced breast cancer. Breast Cancer Res Treat 158: 485-495, 2016.

25. von Minckwitz G, Eidtmann H, Rezai M, Fasching PA, Tesch H, Eggemann H, Schrader I, Kittel K, Hanusch C, Kreienberg R, et al; German Breast Group; Arbeitsgemeinschaft Gynäkologische Onkologie-Breast Study Groups: Neoadjuvant chemotherapy and bevacizumab for HER2-negative breast cancer. N Engl J Med 366: 299-309, 2012.

26. Berry DA and Hudis CA: Neoadjuvant therapy in breast cancer as a basis for drug approval. JAMA Oncol 1: 875-876, 2015. 
27. Mancuso MR, Davis R, Norberg SM, O'Brien S, Sennino B, Nakahara T, Yao VJ, Inai T, Brooks P, Freimark B, et al: Rapid vascular regrowth in tumors after reversal of VEGF inhibition. $\mathrm{J}$ Clin Invest 116: 2610-2621, 2006.

28. Cameron D, Brown J, Dent R, Jackisch C, Mackey J, Pivot X, Steger GG, Suter TM, Toi M, Parmar M, et al: Adjuvant bevacizumab-containing therapy in triple-negative breast cancer (BEATRICE): Primary results of a randomised, phase 3 trial. Lancet Oncol 14: 933-942, 2013.

29. Miller K, O'Neill AM, Dang CT, et al: Bevacizumab in the adjuvant treatment of HER2 negative breast cancer: Final results from Eastern Cooperative Oncology Group E5103. 2014 ASCO Annual Meeting (abstract 500). J Clin Oncol 32 (Suppl 15): 500, 2014.

30. Ulivi P, Scarpi E, Passardi A, Marisi G, Calistri D, Zoli W, Del Re M, Frassineti GL, Tassinari D, Tamberi S, et al: eNOS polymorphisms as predictors of efficacy of bevacizumab-based chemotherapy in metastatic colorectal cancer: Data from a randomized clinical trial. J Transl Med 13: 258, 2015.

31. Lambrechts D, Claes B, Delmar P, Reumers J, Mazzone M, Yesilyurt BT, Devlieger R, Verslype C, Tejpar S, Wildiers H, et al: VEGF pathway genetic variants as biomarkers of treatment outcome with bevacizumab: An analysis of data from the AViTA and AVOREN randomised trials. Lancet Oncol 13: 724-733, 2012.
32. Massuti Sureda B, Jantus-Lewintre E, Gonzalez-Larriba JL, Rodriguez Abreu D, Juan OJ, Domine M, Provencio Pulla M, de Castro J, Camps $\mathrm{C}$ and Rosell R: 37PDSNPS in angiogenic factors as predictive markers for outcome in patients $(\mathrm{P})$ with advanced non-squamous NSCLC (NS-NSCLC) treated with carboplatin, paclitaxel (CP), and bavacizumab (BEV). Final results of angiomet spanish lung cancer group trial. Ann Oncol 26 (Suppl 1): i10, 2015.

33. Mattern J, Koomägi R and Volm M: Association of vascular endothelial growth factor expression with intratumoral microvessel density and tumour cell proliferation in human epidermoid lung carcinoma. Br J Cancer 73: 931-934, 1996.

34. Sikov WM, Barry WT, Hoadley KA, et al: Impact of intrinsic subtype by PAM50 and other gene signatures on pathologic complete response (pCR) rates in triple-negative breast cancer (TNBC) after neoadjuvant chemotherapy (NACT) plus/- carboplatin $(\mathrm{Cb})$ or bevacizumab (Bev): CALGB 40603/150709 (Allianc). Cancer Res 75: S4-S05, 2015. Attribution-NonCommercial-NoDerivatives 4.0 International (CC BY-NC-ND 4.0) License. 